\title{
Heterologous overexpression of Glomerella cingulata FAD-dependent glucose dehydrogenase in Escherichia coli and Pichia pastoris
}

Christoph Sygmund ${ }^{1}$, Petra Staudigl ${ }^{1}$, Miriam Klausberger ${ }^{1}$, Nikos Pinotsis ${ }^{2}$, Kristina Djinović-Carugo ${ }^{2,3}$, Lo Gorton ${ }^{4}$, Dietmar Haltrich ${ }^{1}$ and Roland Ludwig ${ }^{\text {* }^{*}}$

\begin{abstract}
Background: FAD dependent glucose dehydrogenase $(G D H)$ currently raises enormous interest in the field of glucose biosensors. Due to its superior properties such as high turnover rate, substrate specificity and oxygen independence, GDH makes its way into glucose biosensing. The recently discovered GDH from the ascomycete Glomerella cingulata is a novel candidate for such an electrochemical application, but also of interest to study the plant-pathogen interaction of a family of wide-spread, crop destroying fungi. Heterologous expression is a necessity to facilitate the production of GDH for biotechnological applications and to study its physiological role in the outbreak of anthracnose caused by Glomerella (anamorph Colletotrichum) spp.
\end{abstract}

Results: Heterologous expression of active G. cingulata GDH has been achieved in both Escherichia coli and Pichia pastoris, however, the expressed volumetric activity was about 4800-fold higher in P. pastoris. Expression in E. coli resulted mainly in the formation of inclusion bodies and only after co-expression with molecular chaperones enzymatic activity was detected. The fed-batch cultivation of a $P$. pastoris transformant resulted in an expression of $48,000 \mathrm{U} \mathrm{L}^{-1}$ of $\mathrm{GDH}$ activity $\left(57 \mathrm{mg} \mathrm{L}^{-1}\right.$ ). Recombinant $\mathrm{GDH}$ was purified by a two-step purification procedure with a yield of $71 \%$. Comparative characterization of molecular and catalytic properties shows identical features for the GDH expressed in P. pastoris and the wild-type enzyme from its natural fungal source.

Conclusions: The heterologous expression of active GDH was greatly favoured in the eukaryotic host. The efficient expression in P. pastoris facilitates the production of genetically engineered GDH variants for electrochemical-, physiological- and structural studies.

\section{Background}

FAD-dependent glucose dehydrogenase (GDH, EC 1.1.99.10, D-glucose:acceptor 1-oxidoreductase) was first discovered in 1951 in Aspergillus oryzae [1] but remained a relatively little investigated enzyme. In the following decades, only a few FAD-dependent GDHs were characterized from the bacterium Burkholderia cepacia [2], the larvae of the moth Manduca sexta (tobacco hornworm) [3] and the fly Drosophila melanogaster [4]. Since the application of FAD-dependent GDH as electrode catalyst in glucose biosensors [2] and for biofuel cell anodes [5]

\footnotetext{
* Correspondence: roland.ludwig@boku.ac.at

${ }^{1}$ Food Biotechnology Laboratory, Department of Food Sciences and Technology, BOKU-University of Natural Resources and Life Sciences, Muthgasse 18/2 Wien, Austria

Full list of author information is available at the end of the article
}

was published and promoted, more attention was drawn to this enzyme, and new members were identified and characterized, e.g. from the fungi $A$. terreus [6], A. oryzae $[1,7,8]$ and Penicillium lilacinoechinulatum [9]. The advantages of FAD-dependent GDH for their use in glucose biosensors are high turnover rates and a good stability. Moreover, its oxidative half-reaction is unaffected by oxygen, whereas the oxygen turnover in glucose oxidasebased electrodes reduces the electron yield and produces hydrogen peroxide which degrades the biocatalyst. In comparison with pyrroloquinoline quinone (PQQ)dependent GDHs a lower redox potential of FAD-dependent GDH is noteworthy. Two big producers of glucose biosensors, Abbott and Bayer, already implemented FAD-dependent GDHs in some of their products. A novel member of the small family of FAD-dependent 
GDHs was recently discovered in the plant pathogenic fungus Glomerella cingulata (anamorph Colletotrichum gloeosporoides) and characterized [10]. It is an extracellular, glycosylated enzyme showing a narrow substrate specificity with $\beta$-D-glucose and $\mathrm{D}$-xylose as substrates, which are oxidized at the anomeric carbon atom. The electrons are transferred to quinones, phenoxy radicals, redox dyes and iron complexes such as ferricyanide and ferrocenium hexafluorophosphate, but not to molecular oxygen. The biological function of this GDH is still unclear but a role during fungal attack on the host-plant is proposed. By reducing quinones and phenoxy radicals $\mathrm{GDH}$ is able to neutralize the action of plant laccases, phenoloxidases or peroxidases, which are used by infected plant tissues to parry the fungal attack.

Despite the enormous biotechnological relevance of FAD-dependent GDHs there are only scarce reports about their heterologous expression. The catalytic subunit of a bacterial GDH from Burkholderia cepacia was successfully expressed in E. coli. [11]. In contrast, expression levels and productivity for five putative FAD-dependent GDHs from several Aspergillus species in E. coli varied significantly [12]. To our knowledge no eukaryotic expression system was tested and published so far for the expression of FAD-dependent GDHs. We demonstrate that $G$. cingulata GDH (GcGDH) can be heterologously expressed in P. pastoris as well as in E. coli, but with a big difference in the efficiency - expression levels are much higher for the eukaryotic system. In addition, recombinant GDH was compared with the enzyme isolated from its natural source to investigate if their differences in molecular and catalytic properties.

\section{Results}

Expression of $G$. cingulata glucose dehydrogenase in E. coli To evaluate the influence of the N-terminal GcGDH sequence on the amount of soluble, active $G c G D H$ expressed in $E$. coli, three nucleotide sequences coding for GDH with varying $\mathrm{N}$-termini were cloned into pET-21a(+) for expression in E.coli under control of the T7 promoter. Plasmid GC1 encodes the full length GcGDH including its native signal sequence. For plasmid GC2 the nucleotide sequence of the mature protein was cloned right after the start codon, and GC3 contains a truncated version starting 8 amino acids upstream of the FAD binding motif (GXGXXG). The resulting expression vectors were transformed into E. coli expression strains Rosetta 2, T7 Express and T7 Express (pGro7), and cells carrying the plasmids were cultivated in MagicMedia sic! at $20^{\circ} \mathrm{C}$. Cultures were harvested at an optical density at $600 \mathrm{~nm}$ of approximately 15 and disrupted using a French press. The protein concentration of the cleared lysate varied between 6 to $12 \mathrm{mg} \mathrm{mL}^{-1}$. Lysates were tested for GDH activity using the standard DCIP enzyme assay.
Under the tested conditions active GcGDH could only be detected in the T7 expression strains co-transformed with the plasmid pGro7 coding for chaperones. Of the three tested constructs, GC1 showed the highest volumetric activity $\left(10 \mathrm{U} \mathrm{L}^{-1}(\mathrm{DCIP}) ; 5.5 \mathrm{U} \mathrm{L}^{-1}\left(\mathrm{FCPF}_{6}\right)\right)$ in the fermentation medium supplemented with L-arabinose. GDH activity was lower (3.3 DCIP U L' $\mathrm{L}^{-1} 2.0 \mathrm{FcPF}_{6} \mathrm{U} \mathrm{L}^{-1}$ ) for GC2 and no detectable GDH activity was measured for GC3. Activities were around five times lower without arabinose induction of the chaperones. The cell pellet obtained after disruption was tested for the existence of inclusion bodies using SDS-PAGE. The majority of proteins found in the insoluble fraction were of the molecular mass of GcGDH (68 kDa). Refolding experiments were performed with inclusion bodies obtained from the expression experiment yielding the highest amount of soluble GDH. Samples were taken after 1, 12, 24 and $48 \mathrm{~h}$ of incubation in various refolding solutions containing FAD, but no activity could be detected from the tested refolding conditions.

\section{Production and purification of recombinant GcGDH in $P$. pastoris}

The $P$. pastoris expression plasmid pPICGcGDH was constructed by cloning the nucleotide sequence including the native GcGDH signal sequence into the pPIC$\mathrm{ZaA}$ expression vector under control of the methanolinducible AOX promoter. Transformed P. pastoris X-33 cells were checked for integration of the expression cassette into the genome by colony-PCR, and five positive transformants were tested for expression in a smallscale experiment. The best producing clone pPIC-GC1 (2400 $\mathrm{U} \mathrm{L}^{-1} \mathrm{GDH}$ activity) was selected for further studies.

Production of the enzyme was carried out in a 7-liter stirred and aerated bioreactor (Figure 1). The initial glycerol batch phase lasted for $19.5 \mathrm{~h}$ and produced $66.6 \mathrm{~g} \mathrm{~L}^{-1}$ of wet biomass. During the 4 hours of the transition phase from glycerol to methanol the wet biomass further increased up to $99 \mathrm{~g} \mathrm{~L}^{-1}$. At this time a volumetric activity of $1900 \mathrm{U} \mathrm{L}^{-1}$ was already detected. After the transition phase, a methanol feed was started and regulated manually to maintain a steady DO reading of $15 \%$. Levels of wet biomass reached $149 \mathrm{~g} \mathrm{~L}^{-1}$ during this induction phase, and the concentration of soluble protein in the culture supernatant increased from 80 to $300 \mathrm{mg} \mathrm{L}^{-1}$. Volumetric GDH activity in the culture supernatant reached a maximum value of $48,000 \mathrm{U} \mathrm{L}^{-1}$, corresponding to $57 \mathrm{mg}$ of recGcGDH per litre of medium. After $50.5 \mathrm{~h}$ the fermentation was ended since the specific GDH activity in the culture supernatant started to decline.

The recombinant enzyme was purified to homogeneity using a two-step purification protocol employing hydrophobic interaction chromatography and anion exchange 


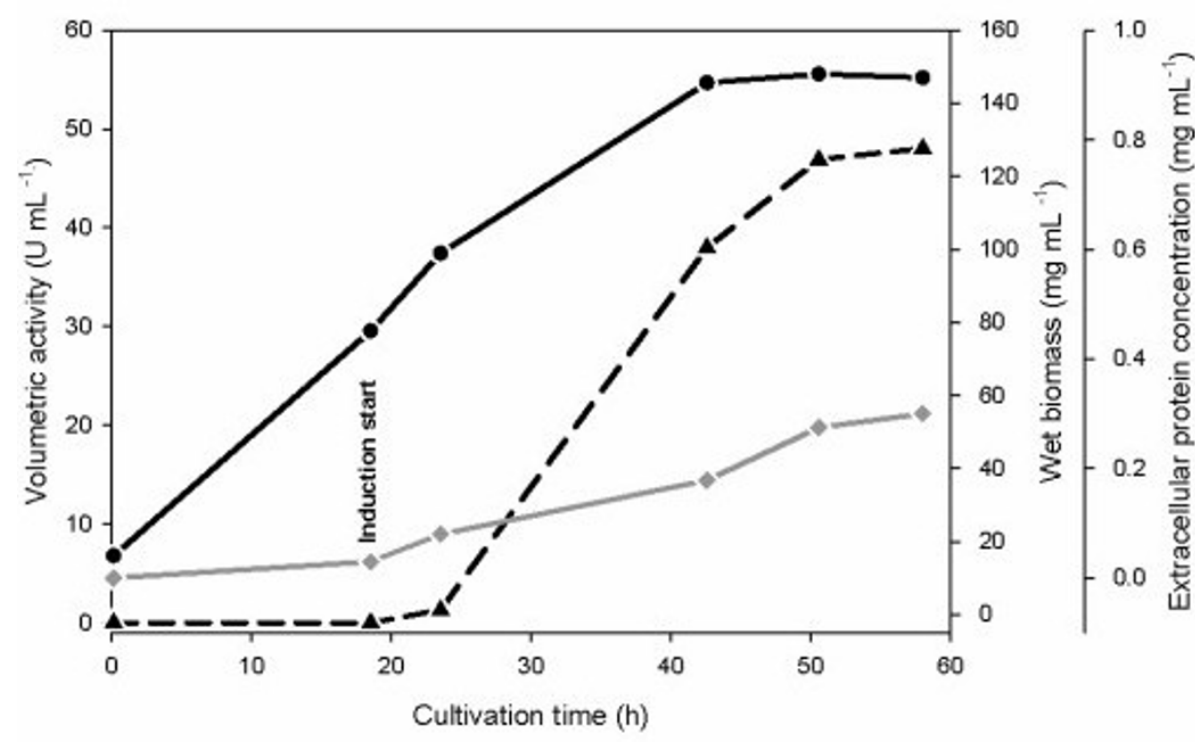

Figure 1 Production of recombinant Glomerella cingulata GDH in P. pastoris. The yeast was cultivated in a 7-L bioreactor. The induction was started by a methanol feed phase. Black circles, wet biomass; black triangles, volumetric activity; grey diamonds, extracellular protein concentration.

chromatography (Table 1). Strict pooling of only the purest fractions resulted in a moderately high yield of 71\%. After purification, a bright-yellow protein solution was obtained and the purity was analyzed by SDSPAGE. The final recombinant GDH preparation had a specific activity of $836 \mathrm{U} \mathrm{mg}^{-1}$.

\section{Molecular and catalytic properties}

The molecular mass of recGcGDH produced in $P$. pastoris was determined by SDS-PAGE, which showed a broad and diffuse band between 88 and $131 \mathrm{kDa}$ (Figure 2). After deglycosylation under denaturing conditions using PNGase F, a single, sharp band with an estimated molecular mass of $67 \mathrm{kDa}$ was obtained. The typical flavoprotein spectrum shows the same characteristics as the spectrum of wild-type $G c \mathrm{GDH}$ with almost identical FAD absorption maxima at 381 and $459 \mathrm{~nm}$ (Figure 3). These peaks disappear upon reduction of the enzyme by adding D-glucose.

The thermal stability of the recGcGDH was preliminary investigated by determining the temperature optimum which was found at $46^{\circ} \mathrm{C}$. For $\mathrm{GDH}$ expressed by G. cingulata (produced according to
[10]) the temperature optimum was $48^{\circ} \mathrm{C}$. In a more detailed investigation using the ThermoFAD technique to derive thermal unfolding transition values (Tm) for different $\mathrm{pH}$ values and buffer substances (Table 2), recGcGDH showed a $\mathrm{pH}$-dependent thermal stability with the highest $\mathrm{Tm}$ values in the acidic range of 4.5 to 6.4. The maximum $\mathrm{Tm}$ value of $56^{\circ} \mathrm{C}$ was measured in $50 \mathrm{mM}$ sodium acetate buffer $\mathrm{pH} 5.0$ and in 50 $\mathrm{mM}$ MES buffer $\mathrm{pH} 5.8$. The activation energy was calculated to be $19.5 \mathrm{~kJ} \mathrm{~mol}^{-1}$ from initial rates in the range of 26 to $51^{\circ} \mathrm{C}$ and is quite similar to the naturally produced GDH $\left(21 \mathrm{~kJ} \mathrm{~mol}^{-1}\right)$.

The kinetic properties of $r e c G c G D H$ were determined for the two best substrates that were identified for wildtype GDH, D-glucose and D-xylose. In these experiments ferrocenium was used as electron acceptor in saturating concentrations. The apparent catalytic constants were determined both at $\mathrm{pH} 5.5$ and 7.5 and compared with those measured for GDH isolated from its natural source G. cingulata (Table 3, [10]). The molecular and catalytic properties of the recombinant enzyme overexpressed in $P$. pastoris are identical to those of the wild-type enzyme.

Table 1 Purification of recombinant Glomerella cingulata glucose dehydrogenase

\begin{tabular}{lccccc}
\hline Purification step & $\begin{array}{c}\text { Total activity } \\
(\mathbf{U})\end{array}$ & $\begin{array}{c}\text { Total protein } \\
(\mathbf{m g})\end{array}$ & $\begin{array}{c}\text { Specific activity } \\
\left(\mathbf{U} \mathbf{~ m g ~}^{-1}\right)\end{array}$ & $\begin{array}{c}\text { Yield } \\
(\%)\end{array}$ & $\begin{array}{c}\text { Purification } \\
\text { (fold) }\end{array}$ \\
\hline Clear supernatant & 215,000 & 1,300 & 165 & 100 & 1 \\
Phenyl-Sepharose & 160,000 & 192 & 833 & 74 & 5 \\
DEAE-Sepharose & 152,000 & 182 & 836 & 71 & 5.1 \\
\hline
\end{tabular}




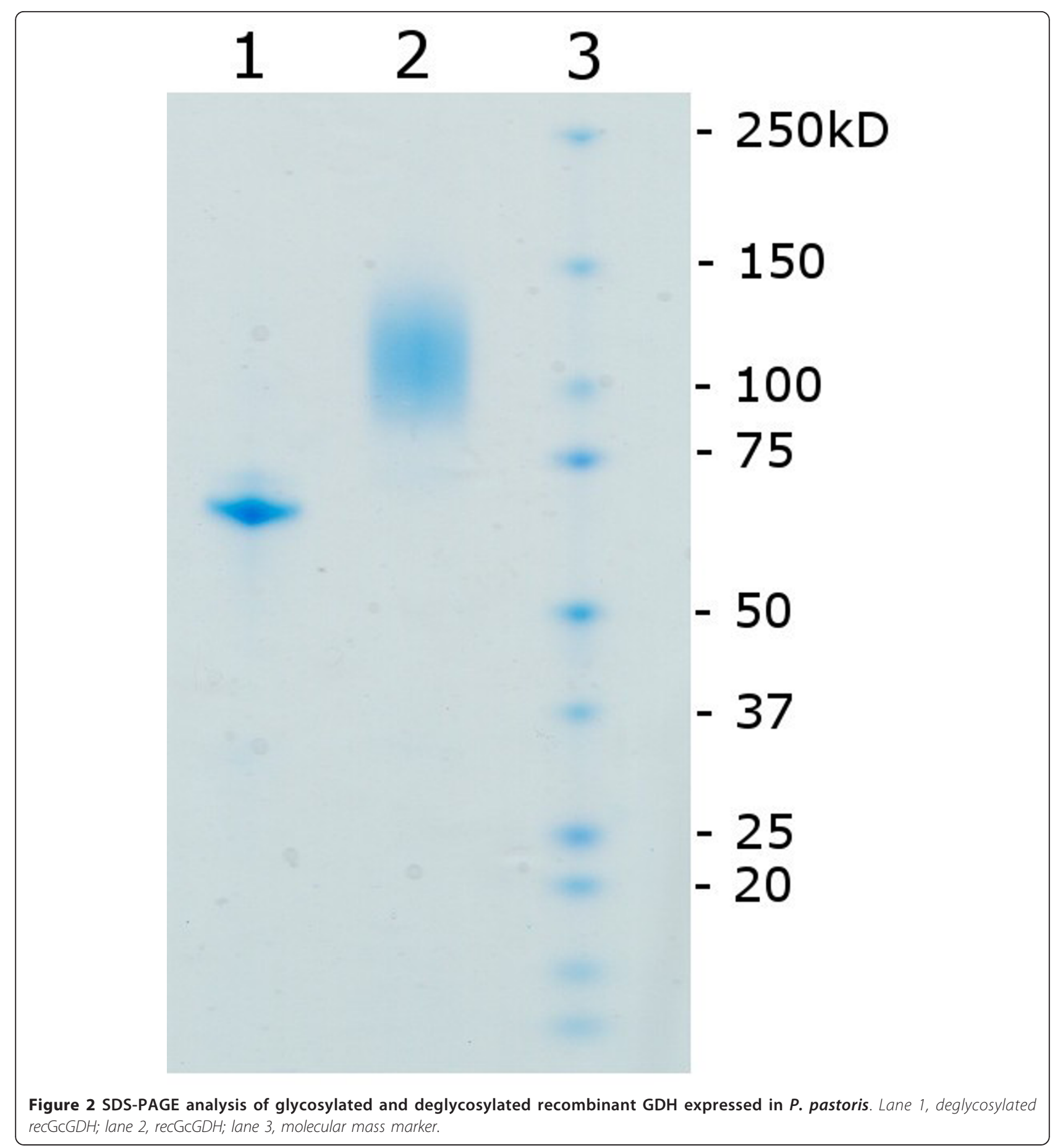

\section{Discussion}

Recently the purification and characterization of a novel FAD-dependent glucose dehydrogenase produced by the plant pathogenic fungus G. cingulata and its proposed role in plant pathogenicity were published [10]. The reported features of this GDH are of interest in two respects: (i) to elucidate the role in the mechanism of plant-pathogen interactions during the infection process and (ii) in electrochemical applications [13,14]. To facilitate biochemical and structural studies as well as engineering of G. cingulata FAD-dependent GDH, the heterologous expression of GcGDH was investigated. To target potential problems with the expression of a heavily glycosylated eukaryotic flavoprotein in a prokaryotic host several approaches were taken. Along with expression of GcGDH with varying N-termini under mild 


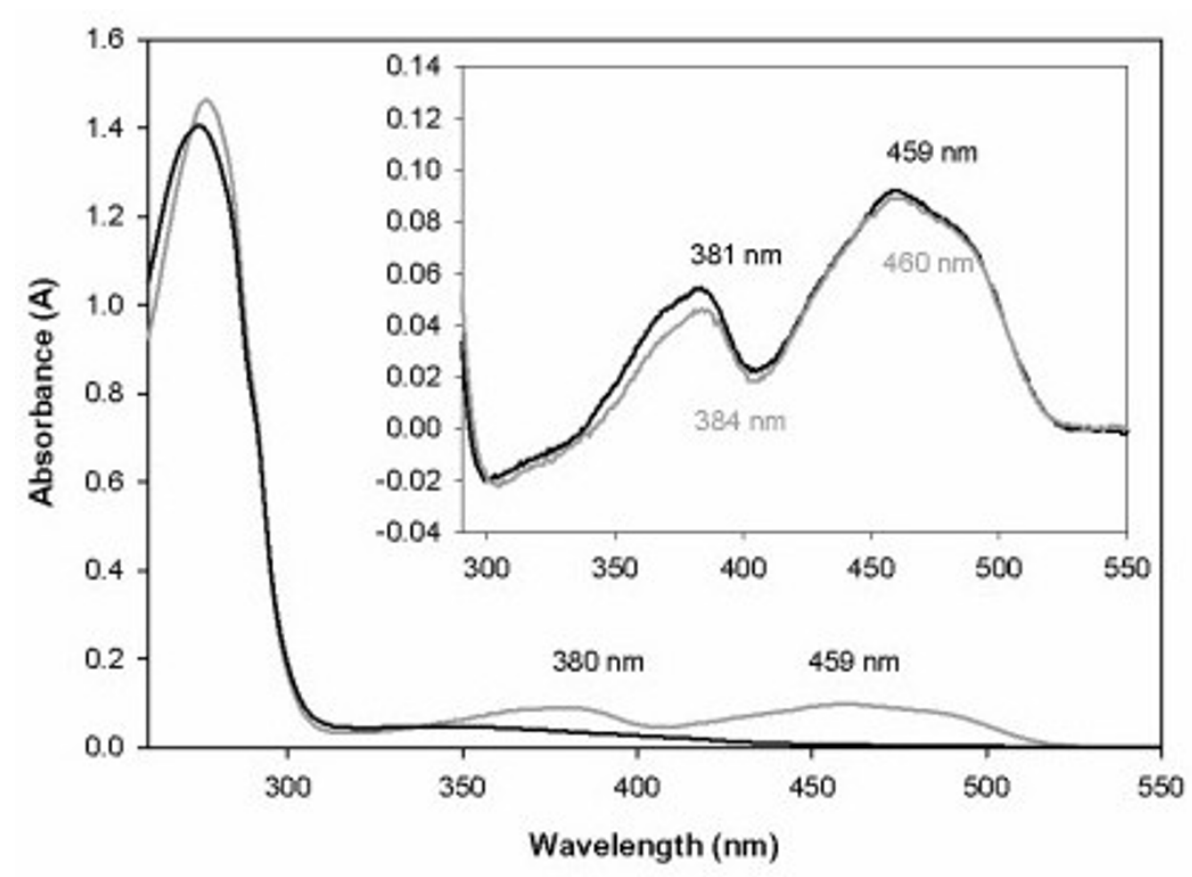

Figure 3 Spectral characterization of GDH showing both the oxidized (gray) and reduced (black) spectra. Glucose was used to reduce the enzyme. The difference spectra (ox-red) of recGDH (black) and wtGDH (gray) are given as inset.

conditions (auto inducing minimal media, $20^{\circ} \mathrm{C}$ ) we also tested different $E$. coli expression strains for their suitability to express soluble and catalytically active GcGDH.

The effect of the $\mathrm{N}$-terminal amino acids on the expression levels of a fungal FAD-dependent GDH in $E$. coli was shown in the US patent 7,741,100 [15]. Expression levels could be increased approximately 10 -fold by deletion of the signal sequence of $A$. oryzae GDH. Therefore, GcGDH was expressed in full length and with the native signal sequence removed. A third, truncated $\mathrm{N}$-terminus was designed according to a sequence alignment of closely related members of the GMC

Table 2 Buffers and pH values used for the analysis of thermal stability (Tm) of G. cingulata GDH using ThermoFAD analysis [23]

\begin{tabular}{lcccccc}
\hline Buffer & $\mathbf{p H}$ & $\mathbf{T m}\left({ }^{\circ} \mathbf{C}\right)$ & $\mathbf{p H}$ & $\mathbf{T m}\left({ }^{\circ} \mathbf{C}\right)$ & $\mathbf{p H}$ & $\mathrm{Tm}\left({ }^{\circ} \mathbf{C}\right)$ \\
\hline Sodium acetate & $\mathbf{4 . 5}$ & 55.0 & $\mathbf{5 . 0}$ & 56.0 & & \\
Sodium citrate & $\mathbf{4 . 7}$ & 55.0 & $\mathbf{5 . 5}$ & 54.0 & & \\
Potassium phosphate & $\mathbf{5 . 0}$ & 55.5 & $\mathbf{6 . 0}$ & 53.5 & $\mathbf{7 . 0}$ & 47.5 \\
Sodium phosphate & $\mathbf{5 . 5}$ & 54.5 & $\mathbf{6 . 5}$ & 51.0 & $\mathbf{7 . 5}$ & 45.0 \\
MES & $\mathbf{5 . 8}$ & 56.0 & $\mathbf{6 . 2}$ & 54.0 & $\mathbf{6 . 5}$ & 53.0 \\
HEPES & $\mathbf{7 . 0}$ & 50.5 & $\mathbf{8 . 0}$ & 43.0 & & \\
Ammonium acetate & $\mathbf{7 . 3}$ & 52.5 & & & & \\
TRIS - HCl & $\mathbf{7 . 5}$ & 47.0 & $\mathbf{8 . 0}$ & 42.5 & $\mathbf{8 . 5}$ & 38.5 \\
Imidazole - HCl & $\mathbf{8 . 0}$ & 41.5 & & & & \\
Bicine - HCl & $\mathbf{8 . 0}$ & 44.0 & $\mathbf{9 . 0}$ & 36.0 & & \\
\hline
\end{tabular}

The buffers were each $50 \mathrm{mM}$. oxidoreductase family. The $\mathrm{N}$-terminal sequences that were successfully used for the expression of $A$. oryzae GDH [15] and the flavin domain of Phanerochaete chrysosporium cellobiose dehydrogenase $(\mathrm{CDH})$ in $E$. coli [16] seem to be highly conserved in these closely related proteins. The analogous sequence MTAYDYIVI was therefore chosen as N-terminal sequence for the third variant of GcGDH. Surprisingly, although in a prokaryotic expression host, expression levels of GcGDH were highest with the full-length protein, which included its own signal sequence. For the variant lacking the signal sequence the volumetric activity decreased three-fold, and no activity was detected for the third and shortest construct. For all tested expression constructs the fraction of GDH protein found in inclusion bodies (as judged by SDS-PAGE) was high. For the rather closely related $P$. amagasakiense glucose oxidase (GOX) refolding experiments from inclusion bodies were successful, retrieving $\sim 10 \%$ of the totally aggregated GOX in an active form [17]. Although the same or slightly modified conditions were used, the same result could not be reproduced for GcGDH. We conclude, that although GOx is the phylogenetically closest relative of GDH [10], the structure of GDH is different enough not to favour cofactor reconstitution under the same or similar conditions.

In addition to in vitro refolding of incorrectly folded protein several other methods have been described in 
Table 3 Apparent kinetic constants of recombinant and wild-type Glomerella cingulata GDH for either D-glucose or D-xylose as substrate, with the concentration of the electron acceptor ferrocenium ion held constant at $20 \mu \mathrm{M}$

\begin{tabular}{lcccc}
\hline Substrate and $\mathbf{p H}$ & enzyme & $\begin{array}{c}\mathbf{K}_{\mathbf{m}} \\
(\mathbf{m M})\end{array}$ & $\begin{array}{c}\mathbf{k}_{\mathbf{c a t}} \\
\left(\mathbf{s}^{-1}\right)\end{array}$ & $\begin{array}{c}\mathbf{k}_{\text {cat }} / \mathbf{K}_{\mathbf{m}} \\
\left(\mathbf{M}^{-1} \mathbf{s}^{-1}\right)\end{array}$ \\
\hline Glucose, $\mathrm{pH}=5.5$ & $\mathrm{wt}$ & $10.2 \pm 0.2$ & $180 \pm 3$ & $17.6 \times 10^{3}$ \\
& rec & $10.1 \pm 0.4$ & $179 \pm 4$ & $17.7 \times 10^{3}$ \\
\hline Glucose, $\mathrm{pH}=7.5$ & $\mathrm{wt}$ & $19.0 \pm 0.3$ & $380 \pm 6$ & $20.0 \times 10^{3}$ \\
& rec & $17.1 \pm 0.7$ & $418 \pm 4$ & $24.5 \times 10^{3}$ \\
\hline Xylose, $\mathrm{pH}=5.5$ & $\mathrm{wt}$ & $21 \pm 0.6$ & $40 \pm 1.5$ & $1.90 \times 10^{3}$ \\
& rec & $26 \pm 2.7$ & $63 \pm 1.9$ & $2 \times 10^{3}$ \\
\hline Xylose, $\mathrm{pH}=7.5$ & wt & $24 \pm 1.5$ & $60 \pm 2$ & $2.5 \times 10^{3}$ \\
& rec & $23 \pm 0.7$ & $61 \pm 1$ & $2.7 \times 10^{3}$ \\
\hline
\end{tabular}

Kinetic data were determined at $30^{\circ} \mathrm{C}$, the data for wild-type $\mathrm{GCGDH}$ are from [10].

literature for promoting the synthesis of active recombinant protein in the soluble cytoplasmic fraction rather than as inclusion bodies $[18,19]$. Increased amounts of the chaperone system GroEL/GroES in the cytoplasm apparently reduces the accumulation of aggregated GcGDH in the cell, leading to small amounts of active soluble GcGDH. The supply of tRNAs for 7 rare codons by the strain Rosetta 2, showed no beneficial effect on the expression of GcGDH. This, however, was to be expected since codon analysis of the gcgdh gene revealed no sequences that could affect the transcriptional or translational efficiencies.

A further strategy to reduce the in vivo aggregation of recombinant $G c G D H$ in $E$. coli was to use slow growth and weak inducing conditions. To this end, the cultivation temperature was lowered to $20^{\circ} \mathrm{C}$ and an auto-inducing medium (MagicMedia) was used. It was shown previously that yields of a target protein as well as cell mass can be increased substantially by using such mild conditions [20]. Cell densities were increased up to $30 \mathrm{~g}$ $\mathrm{L}^{-1}$ compared to $10 \mathrm{~g} \mathrm{~L}^{-1}$ obtained by the standard LB medium. Even though all these considerations were taken into account for the expression of GcGDH in $E$. coli a volumetric activity of $10 \mathrm{U} \mathrm{L}^{-1}$ could be produced under optimized conditions. Since expression rates in $P$. pastoris were much higher no effort was made to purify GcGDH from E. coli cultures.

When using the eukaryotic expression system, GcGDH could be expressed extracellularly in high yields using the native signal sequence, which indicates that this signal sequence is properly recognized and processed by the yeast. A final volumetric activity of 48,000 $\mathrm{U} \mathrm{L}^{-1}$ and a space-time yield of $24 \mathrm{mg} \mathrm{L}^{-1} \mathrm{~d}^{-1}$ could be achieved by $P$. pastoris. This is a 70-fold improvement of the space-time yield compared to the wild-type producer. The cultivation yielded a total of $57 \mathrm{mg} \mathrm{L}^{-1}$ of recombinant protein, which corresponds to $\sim 20 \%$ of total extracellular protein. The purification protocol resulted in a protein preparation of high purity (as checked by SDS-PAGE) with a specific activity of $836 \mathrm{U} \mathrm{mg}^{-1}$, which is comparable to the wild type preparation $\left(840 \mathrm{U} \mathrm{mg}^{-1},[10]\right)$. Since the first purification step already yielded a protein of high specific activity $\left(833 \mathrm{U} \mathrm{mg}^{-1}\right)$ the procedure might be reduced to a one-step purification. All (bio)physical and catalytic properties studied for recGcGDH are essentially identical to those of the wild-type enzyme isolated from the original source G. cingulata (Table 3, [10]). The high degree of glycosylation of recombinant GcGDH (approx. $65 \%$ as judged from SDS-PAGE, Figure 2) is also found in native GcGDH (approx. 70\%, [10]). These values are certainly an overestimation by SDS-PAGE, which is known to smear bands of glycosylated proteins, but the range of the bands of native $(95-135 \mathrm{kDa})$ and recombinant $(88-131 \mathrm{kDa}) \mathrm{GcGDH}$ are nearly identical. The temperature optimum for recGcGDH is $46^{\circ} \mathrm{C}$ and close to the previously reported value for an $\mathrm{FAD}$-dependent glucose dehydrogenase from $A$. terreus $\left(50^{\circ} \mathrm{C}\right)$ [14].

This study reports and compares the successful heterologous expression of Glomerella cingulata GDH in P. pastoris and E. coli. The glycosylation of this protein seems to play an important role for folding into the correct conformation, as already shown for other proteins as well [21]. This makes the eukaryotic host more suitable for the production of recGcGDH, which displays properties that are essentially identical to those of the wild-type enzyme [10]. The expression in E. coli has the advantage that glycosylation-free GcGDH can be obtained, which is useful for e.g. crystallization studies. However, for this application the production in the prokaryotic host has to be optimized further to provide sufficient amounts of protein.

\section{Conclusions}

The suitability of a eukaryotic and a prokaryotic expression system for the heterologous overexpression of an extracellular fungal glucose dehydrogenase is tested by this study. The expression of GcGDH in P. pastoris provides a suitable method for the easy preparation of 
sufficient amounts of GDH as well as genetically engineered GDH variants for further applications in electrochemistry, for structure/function studies or for the study of plant-pathogen interactions of this attractive novel enzyme.

\section{Methods}

\section{Strains and media}

P. pastoris X-33 is a component of the EasySelect Pichia Expression Kit and was obtained from Invitrogen. Chemical competent E. coli strain NEB 5-alpha was purchased from New England Biolabs (NEB) and used for maintenance and propagation of plasmids. E. coli expression strains Rosetta 2 and T7 Express were ordered from Novagen and from New England Biolabs, respectively. $E$. coli cells were cultivated in LB-medium (peptone from casein $10 \mathrm{~g} \mathrm{~L}^{-1}$, yeast extract $5 \mathrm{~g} \mathrm{~L}^{-1}$, $\mathrm{NaCl} 10 \mathrm{~g} \mathrm{~L}^{-1}$ ) containing $100 \mathrm{mg} \mathrm{L}^{-1}$ ampicillin and/or $30 \mathrm{mg} \mathrm{L}^{-1}$ chloramphenicol. Low Salt LB-medium $(\mathrm{NaCl}$ reduced to $\left.5 \mathrm{~g} \mathrm{~L}^{-1}\right)$ was used when zeocin $\left(25 \mathrm{mg} \mathrm{L}^{-1}\right)$ was used as selection marker. MagicMedia sic! E. coli expression medium (Invitrogen) was used for expression studies in E. coli. P. pastoris transformants were grown on YPD plates (yeast extract $10 \mathrm{~g} \mathrm{~L}^{-1}$, peptone $20 \mathrm{~g} \mathrm{~L}^{-1}$, dextrose $10 \mathrm{~g} \mathrm{~L}^{-1}$, zeocin $100 \mathrm{mg} \mathrm{L}^{-1}$ ) and the Basal Salts Medium (Invitrogen) was used for fermentation.

\section{Chemicals and Vectors}

All chemicals were purchased from Sigma, Fluka, Roth or VWR and were of the highest purity available. Primers were from VBC-Biotech and nucleotide sequences are shown in Table 4. Restriction enzymes and T4-ligase were purchased from Fermentas, Phusion polymerase from NEB and the yeast expression vector pPICZ $\alpha \mathrm{A}$ from Invitrogen. The plasmid pET-21a(+)from Novagen was used for expression in E. coli. Plasmid pGro7 encoding the chaperones GroEL and GroES was purchased from TAKARA Bio Inc. (Japan).

\section{Heterologous expression in E. coli}

The published plasmid pGC1 [10] was used as template for the amplification of GcGDH cDNA (JF731352) with three different forward primers (GC-GDHndeIfw1 - 3) and the reverse primers GC-GDHnotIrv1. The three

\section{Table 4 Nucleotide sequences of primers}

\begin{tabular}{lc}
\hline Primer name & Sequence (from $\mathbf{5}^{\prime}$ to $\mathbf{3}^{\prime}$ ) \\
\hline GC-GDHndelfw1 & TATCATATGAAGAACCTCATTCCTC \\
GC-GDHndelfw2 & TATCATATGCCAGGTCTGCCCCCAGGG \\
GC-GDHndelfw3 & TATCATATGACGGCATACGACTATATTGTC \\
GC-GDHnotlrv & ATACGGCGTCATTAAGCAGCAGCCTTGATCAGAT \\
GC-GDH-BstBI+SS & TATTCGAAATGAAGAACCTCATTCCTCTTCC \\
GC-seq-rv1 & AGGTAGAAGCACCACCAGAGG \\
\hline
\end{tabular}

resulting nucleotide sequences encoded GcGDH with varying $\mathrm{N}$-termini. Both the PCR fragments and the expression vector pET-21a(+) were digested with $\mathrm{NdeI}$ and Not $\mathrm{I}$ and ligated using the Rapid DNA Ligation Kit from Fermentas. Correct insertion of the genes and the absence of mutations were checked by DNA sequencing and verified plasmids were transformed into $E$. coli Rosetta 2, E. coli T7 Express and E. coli T7 Express carrying the plasmid pGro7. In order to compare the expression levels of GcGDH with these 9 different expression strategies, small-scale cultivation in $125-\mathrm{mL}$ baffled shaken flasks filled with $30 \mathrm{~mL}$ media were performed at $20^{\circ} \mathrm{C}$. To reduce time-consuming steps such as monitoring optical density (OD) prior to induction or adding appropriate inducers, the autoinducing MagicMedia (Invitrogen) was used for this comparative study. Chaperone co-expression was tested both with $1 \mathrm{mg}$ $\mathrm{mL}^{-1} \mathrm{~L}$-arabinose for induction and without added inducer.

All cultures were grown at $37^{\circ} \mathrm{C}$ for $5 \mathrm{~h}$ and then further cultivated overnight at $20^{\circ} \mathrm{C}$. Cell suspensions were centrifuged at $4000 \times g$ for $10 \mathrm{~min}$ at $4^{\circ} \mathrm{C}$, the cell pellets were suspended in lysis buffer $(50 \mathrm{mM}$ potassium phosphate buffer pH 6.5 supplemented with $5.7 \mathrm{mM}$ PMSF), and disrupted by using a French Press. The crude extract was cleared by centrifugation $(4000 \times g, 30 \mathrm{~min}$, $4^{\circ} \mathrm{C}$ ), the supernatant was tested for GDH activity by the colorimetric DCIP assay, and the pellet was analyzed for insoluble GDH by SDS-PAGE. Refolding experiments were done according to the protocol of the Renaturation Basic Kit for Proteins (Sigma). Additionally, flavin adenine dinucletide (FAD) was added to the renaturing solution at a concentration of $50 \mu \mathrm{M}$.

\section{Heterologous expression in Pichia pastoris}

$G c G D H$-encoding cDNA was amplified using the primers GC-GDH-BstBI+SS and GC-GDH-NotI. The PCR amplicon was digested with Bsp119I and NotI and cloned into the yeast expression vector pPICZ $\alpha$ A. The resulting plasmid pPICGcGDH was linearized with MssI and transformed into electrocompetent $P$. pastoris X-33 cells prepared according to the operating instructions and applications guide of the MicroPulser electroporation apparatus (Biorad). Transformants were selected on YPD zeocin plates, and the integration of the gene was checked by colony PCR with the primers AOX-fw and GC-seq-rv1. Five positive colonies were selected for expression studies in baffled shaken flasks. Pre-cultures $(50 \mathrm{~mL})$ were grown overnight at $30^{\circ} \mathrm{C}$ in YPD medium containing $50 \mathrm{mg} \mathrm{L}^{-1}$ zeocin. After approximately $16 \mathrm{~h}$ of growth the pre-cultures were transferred into 1-L baffled shaken flasks containing $300 \mathrm{~mL}$ of BMMY medium. Methanol $(0.5 \% \mathrm{v} / \mathrm{v}$ final concentration) was added regularly (approximately every $12 \mathrm{~h}$ ) while incubating at $30^{\circ} \mathrm{C}$ 
and shaking at $150 \mathrm{rpm}$. Samples were taken every day and analyzed for protein concentration and GDH activity.

\section{Enzyme production and purification}

Recombinant $G c \mathrm{GDH}$ was produced in a 7-L glass vessel fermenter (MBR) filled with $4 \mathrm{~L}$ of medium (Basal Salts Medium). After autoclaving, the $\mathrm{pH}$ of the medium was adjusted to 5.0 with $28 \%$ ammonium hydroxide and maintained at this $\mathrm{pH}$ for the entire fermentation process. The fermentation was started by adding $0.4 \mathrm{~L}(9 \%$ $\mathrm{v} / \mathrm{v}$ ) of preculture grown on YPD medium in 1-L baffled shaken flasks at $125 \mathrm{rpm}$ and $30^{\circ} \mathrm{C}$ overnight. The cultivation was executed according to the Pichia Fermentation Guideline of Invitrogen and enzyme production was induced with methanol. At the transition phase from glycerol to methanol feed the protocol was altered according to Zhang et al. [22]. At the end of the glycerol batch phase methanol $(0.2 \% \mathrm{v} / \mathrm{v})$ was injected aseptically into the fermenter, and the glycerol feed faded out by a linear ramp $20 \mathrm{~g} \mathrm{~L}^{-1} \mathrm{~h}^{-1}$ to $0 \mathrm{~g} \mathrm{~L}^{-1} \mathrm{~h}^{-1}$ over $4 \mathrm{~h}$. Once the dissolved oxygen concentration spiked, the methanol feed was started. It was regulated to keep a stable dissolved oxygen concentration of $15 \%$. The cultivation temperature was $30^{\circ} \mathrm{C}$, the variable airflow rate was around $6 \mathrm{~L}$ $\mathrm{min}^{-1}$, and the agitation was set to $800 \mathrm{rpm}$. Samples were taken regularly and clarified by centrifugation. The pellet was used to determine wet biomass. GDH activity and extracellular protein concentration were assayed in the supernatant.

The fermentation broth was clarified by centrifugation $\left(6000 \times \mathrm{g} ; 30 \mathrm{~min} ; 4^{\circ} \mathrm{C}\right)$ and saturated ammonium sulfate solution was slowly added to give a $60 \%$ saturated solution. Precipitates were removed by ultracentrifugation $\left(30,000 \times \mathrm{g} ; 15 \mathrm{~min} ; 4^{\circ} \mathrm{C}\right)$ and the enzyme was purified by hydrophobic interaction chromatography on a 400-mL PHE Sepharose 6 fast flow column (chromatographic equipment and materials from GE Healthcare Biosciences) equilibrated with $50 \mathrm{mM}$ phosphate buffer pH 7 containing 60\% (saturation) ammonium sulfate. Proteins were eluted within a linear gradient from 60 to $0 \%$ ammonium sulfate in 8.5 column volumes $(\mathrm{CV}, 3.4$ $\mathrm{L})$ and collected in $50 \mathrm{~mL}$ fractions. Active fractions were pooled and diafiltrated using a hollow fiber crossflow module (Microza UF module SLP-1053, $10 \mathrm{kDa}$ cut-off, Pall Corporation). The partially deionized enzyme solution $\left.(3 \mathrm{mS} \mathrm{cm})^{-1}\right)$ was applied to a column packed with $100 \mathrm{~mL}$ DEAE-Sepharose FF, previously equilibrated with $50 \mathrm{mM}$ phosphate buffer, $\mathrm{pH}$ 7.5. Proteins were eluted within a linear salt gradient from 0 to $2 \mathrm{M} \mathrm{NaCl}$ in $10 \mathrm{CV}(1 \mathrm{~L})$. The pooled fractions were concentrated and the buffer was exchanged by diafiltration to $50 \mathrm{mM} \mathrm{MES} \mathrm{pH} \mathrm{5.8,} \mathrm{and} \mathrm{the} \mathrm{enzyme} \mathrm{solution}$ was filter sterilized, aliquoted and stored at $-30^{\circ} \mathrm{C}$.

\section{Enzyme assays and protein determination}

Glucose dehydrogenase activity was assayed spectrophotometrically using 2,6-dichloroindophenol (DCIP, $\varepsilon_{520}=$ $6.9 \mathrm{mM}^{-1} \mathrm{~cm}^{-1}$ ) as electron acceptor. The reaction was followed for $180 \mathrm{~s}$ at $30^{\circ} \mathrm{C}$ in a Lambda $35 \mathrm{UV} / \mathrm{Vis}$ spectrophotometer (Perkin Elmer). The DCIP-based assay contained (final concentrations) $50 \mathrm{mM}$ sodium acetate buffer, pH 5.5, $300 \mu \mathrm{M}$ DCIP and $100 \mathrm{mM}$ D-glucose. Alternatively, ferrocenium hexafluorophosphate $\left(\varepsilon_{300}=\right.$ $4.3 \mathrm{mM}^{-1} \mathrm{~cm}^{-1}$ ) was used as electron acceptor for the determination of the catalytic constants to enable measurements in the range of $\mathrm{pH} 5.5$ and 7.5. One unit of GDH activity was defined as the amount of enzyme necessary for the reduction of $1 \mu \mathrm{mol}$ glucose or electron acceptor per min under the assay conditions [10]. It is noted that DCIP is a two-electron acceptor, but the ferrocenium ion a one-electron acceptor. The protein concentration was determined by the method of Bradford using a prefabricated assay (Bio-Rad) and bovine serum albumin as standard.

\section{Molecular properties}

SDS-PAGE was carried out using Mini-PROTEAN TGX precast gels with a denaturing gradient of 4-15\%. Protein bands were visualized by staining with Bio-Safe Coomassie (Bio-Rad). Dual Color Precision Plus Protein Standard (Bio-Rad) was used for mass determination. All procedures were done according to the manufacturer's recommendations. To estimate the degree of glycosylation homogenous recGcGDH was treated with PNGase F (NEB) under denaturing conditions according to the manufacturer's instructions. The spectrum of homogeneously purified recGcGDH was recorded at room temperature from 250 to $550 \mathrm{~nm}$ in both the oxidized and reduced state using a U-3000 Hitachi spectrometer (Tokyo, Japan). GDH was diluted in $50 \mathrm{mM}$ citrate buffer, pH 5.5 to an absorbance of $\sim 1.5$ at $280 \mathrm{~nm}$ and the spectrum was recorded before and shortly after the addition of glucose to the cuvette. The temperature profile of activity for wildtype and recombinant GDH was determined in parallel by measuring the average GDH activity over $5 \mathrm{~min}$ from 25 to $62^{\circ} \mathrm{C}$ in temperature controlled DCIP assays.

\section{ThermoFAD analysis}

The Thermofluor-based ThermoFAD method [23] was used to monitor protein unfolding for analysis of thermal stability of recGcGDH in a set of 22 different buffers, each at $50 \mathrm{mM}$, over a $\mathrm{pH}$ range from $\mathrm{pH}$ 4.5-9.0. Buffers used can be seen in Table 4. The method takes advantage of the intrinsic fluorescence of the FAD cofactor, and does not depend on fluorescent dyes. recGcGDH was diluted in buffer to a final concentration 
of $1 \mathrm{mg} \mathrm{mL} \mathrm{m}^{-1}$ and subsequently analyzed in triplicates in $50 \mu \mathrm{L}$ aliquots per well. A real-time PCR cycler (iCycler, Bio-Rad) providing a MyiQ Optics Module, and SYBR-Green filters (523-543 nm) was used to record the signals. The samples were heated in $0.5^{\circ} \mathrm{C}$ steps $(20$ s per step) from $30^{\circ}$ to $95^{\circ} \mathrm{C}$. The fluorescence signal was measured at the end of each step.

\section{Steady-state kinetics}

Apparent kinetic constants for D-glucose and D-xylose were determined with ferrocenium hexafluorophosphate as electron acceptor at a fixed concentration of $200 \mu \mathrm{M}$ using glucose in the range of 1-100 $\mathrm{mM}$, and xylose in the range of 100-1500 mM. Constants were calculated using nonlinear least-squares regression by fitting the observed data to the Michaelis-Menten equation (Sigma Plot 11, Systat Software).

\section{Acknowledgements}

The authors thank the Austrian Academy of Science (APART project 11322), the European Commission (FP7 project 3D-Nanobiodevice NMP4-SL-2009229255) and the Federal Ministry of Economy, Family and Youth through "Laura Bassi Centre of Expertise" initiative project Number 253275 for financial support.

\section{Author details}

${ }^{1}$ Food Biotechnology Laboratory, Department of Food Sciences and Technology, BOKU-University of Natural Resources and Life Sciences, Muthgasse 18/2 Wien, Austria. ${ }^{2}$ Department of Structural and Computational Biology, Max F. Perutz Laboratories, University of Vienna, Campus Vienna Biocenter 5, A-1030 Vienna, Austria. ${ }^{3}$ Department of Biochemistry, Faculty of Chemistry and Chemical Technology, University of Ljubljana, Aškerčeva 5, 1000 Ljubljana, Slovenia. ${ }^{4}$ Department of Biochemistry and Structural Biology, Lund University, P. O. Box 124, 22100 Lund, Sweden.

\section{Authors' contributions}

$\mathrm{CS}$ and $\mathrm{RL}$ drafted the outline of the expression experiments, protein purification and characterization. PS and NP carried out the construction of the expression vectors and PS performed E. coli expression studies. MK conducted the $P$. pastoris fermentation, GDH purification and characterization. NP and KDj-C helped with the selection of expression vectors, strains and cultivation conditions and participated in stability studies. LG suggested stability experiments and interpreted the data. CS wrote the first draft of the manuscript. KDj-C and LG revised the manuscript. $\mathrm{RL}$ and $\mathrm{DH}$ coordinated the study, verified and interpreted results and revised the final manuscript. All authors have read and approved the final manuscript.

\section{Competing interests}

The authors declare that they have no competing interests.

Received: 19 September 2011 Accepted: 12 December 2011 Published: 12 December 2011

\section{References}

1. Ogura Y: Studies on the glucose dehydrogenase of Aspergillus oryzae. J Biochem 1951, 38(1):75-84.

2. Sode K, Tsugawa W, Yamazaki T, Watanabe M, Ogasawara N, Tanaka M: A novel thermostable glucose dehydrogenase varying temperature properties by altering its quaternary structures. Enzyme Microb Technol 1996, 19(2):82-85.

3. Lovallo N, Cox-Foster DL: Alteration in FAD-glucose dehydrogenase activity and hemocyte behavior contribute to initial disruption of
Manduca sexta immune response to Cotesia congregata parasitoids. $J$ Insect Physiol 1999, 45(12):1037-1048.

4. Cavener DR, Maclntyre RJ: Biphasic expression and function of glucose dehydrogenase in Drosophila melanogaster. Proc Natl Acad Sci USA 1983 80(20 l):6286-6288

5. Okuda-Shimazaki J, Kakehi N, Yamazaki T, Tomiyama M, Sode K: Biofuel cell system employing thermostable glucose dehydrogenase. Biotechnol Lett 2008, 30(10):1753-1758.

6. Omura H, Sanada H, Yada T, Morita T, Kuyama M, Ikeda T, Kano K, Tsujimura S: Coenzyme-binding glucose dehydrogenase. 2004, In EP 1 584675.

7. Bak T-G, Sato R: Studies on the glucose dehydrogenase of Aspergillus oryzae: I. Induction of its synthesis by p-benzoquinone and hydroquinone. Biochim Biophys Acta, Enzymol 1967, 139(2):265-276.

8. Tsuji Y, Kitabayashi M, Kishimoto T, Nishiya Y: Glucose dehydrogenase from Aspergillus oryzae. 2010, In US patent 7,655,130 B2.

9. Aiba H, Tsugura-shi J: Novel glucose dehydrogenase. US Patent 2007/ 0105174.

10. Sygmund C, Klausberger M, Felice A, Ludwig R: Reduction of quinones and phenoxy radicals by extracellular glucose dehydrogenase from Glomerella cingulata suggests a role in plant pathogenicity. Microbiology 2011, 157(11):3203-3212.

11. Inose K, Fujikawa M, Yamazaki T, Kojima K, Sode K: Cloning and expression of the gene encoding catalytic subunit of thermostable glucose dehydrogenase from Burkholderia cepacia in Escherichia coli. Biochim Biophys Acta - Proteins \& Proteomics 2003, 1645(2):133-138.

12. Mori K, Nakajima M, Kojima K, Murakami K, Ferri S, Sode K: Screening of Aspergillus-derived FAD-glucose dehydrogenases from fungal genome database. Biotechnology Letters 1-9.

13. Heller A, Feldman B: Electrochemical glucose sensors and their applications in diabetes management. Chemical Reviews 2008, 108(7):2482-2505

14. Tsujimura S, Kojima S, Kano K, Ikeda T, Sato M, Sanada H, Omura H: Novel FAD-Dependent Glucose Dehydrogenase for a Dioxygen-Insensitive Glucose Biosensor. Biosci, Biotechnol, Biochem 2006, 70(3):654-659.

15. Kitabayashi M, Tsuji Y, Kawarabayshi Y, Kishimoto T, Nishiya Y: Method for highly expressing recombinant glucose dehydrogenase derived from filamentous fungi. 2010.

16. Desriani Ferri S, Sode K: Functional expression of Phanerochaete chrysosporium cellobiose dehydrogenase flavin domain in Escherichia coli. Biotechnology Letters 32(6):855-859.

17. Witt $S$, Singh M, Kalisz HM: Structural and kinetic properties of nonglycosylated recombinant Penicillium amagasakiense glucose oxidase expressed in Escherichia coli. Applied and Environmental Microbiology 1998, 64(4):1405-1411.

18. Sørensen $H$, Mortensen $\mathrm{K}$ : Soluble expression of recombinant proteins in the cytoplasm of Escherichia coli. 2005, 4:1

19. Sørensen HP, Mortensen KK: Advanced genetic strategies for recombinant protein expression in Escherichia coli. Journal of Biotechnology 2005, 115(2):113-128.

20. Studier FW: Protein production by auto-induction in high density shaking cultures. Protein expression and purification 2005, 41(1):207-234.

21. Shental-Bechor D, Levy Y: Effect of glycosylation on protein folding: $A$ close look at thermodynamic stabilization. Proceedings of the National Academy of Sciences of the United States of America 2008, 105(24):8256-8261.

22. Zhang $W$, Inan $M$, Meagher MM: Fermentation strategies for recombinant protein expression in the methylotrophic yeast Pichia pastoris. Biotechnology and Bioprocess Engineering 2000, 5(4):275-287.

23. Forneris F, Orru R, Bonivento D, Chiarelli LR, Mattevi A: ThermoFAD, a Thermofluor ${ }^{\oplus}$-adapted flavin ad hoc detection system for protein folding and ligand binding. FEBS Journal 2009, 276(10):2833-2840.

doi:10.1186/1475-2859-10-106

Cite this article as: Sygmund et al:: Heterologous overexpression of Glomerella cingulata FAD-dependent glucose dehydrogenase in Escherichia coli and Pichia pastoris. Microbial Cell Factories 2011 10:106. 